\title{
Comparison of the Rose Bengal Plate and the Complement Fixation Tests with the Tube Agglutination Test for Diagnosis of Human Brucellosis
}

\author{
Ying-Hock Teng ${ }^{1,2}$, Jung-Jing Teng ${ }^{3}$, Samantha Chao ${ }^{4}$, Hsu Chao ${ }^{5}$, Suryakant D. Waghela ${ }^{6,7}$ \\ ${ }^{1}$ Department of Emergency Medicine, School of Medicine, Chung Shan Medical University, Taiwan \\ ${ }^{2}$ Department of Emergency Medicine, Chung Shan Medical University Hospital, Taiwan \\ ${ }^{3}$ Residency Program, Civil Aviation General Hospital, Peking University Health Science Center, Beijing, China \\ ${ }^{4}$ Rice University, Houston, TX, USA \\ ${ }^{5}$ HGSC, Baylor College of Medicine, Houston, TX, USA \\ ${ }^{6}$ Veterinary Research Laboratory, Kabete, Kenya \\ ${ }^{7}$ Department of Veterinary Pathobiology, Texas A\&M University, College Station, TX, USA \\ Email: *cshy392@csh.org.tw
}

How to cite this paper: Teng, Y.-H., Teng, J.-J., Chao, S., Chao, H. and Waghela, S.D. (2017) Comparison of the Rose Bengal Plate and the Complement Fixation Tests with the Tube Agglutination Test for Diagnosis of Human Brucellosis. Open Journal of Clinical Diagnostics, 7, 73-82.

https://doi.org/10.4236/ojcd.2017.73008

Received: June 10, 2017

Accepted: July 25, 2017

Published: July 28, 2017

Copyright $(9) 2017$ by authors and Scientific Research Publishing Inc. This work is licensed under the Creative Commons Attribution International License (CC BY 4.0).

http://creativecommons.org/licenses/by/4.0/

\begin{abstract}
Brucellosis is a zoonotic disease of economic importance. The clinical manifestations vary in humans; therefore a good diagnostic test is required to confirm the disease. The serum tube agglutination (SA) test, though still the most widely test used, can be problematic for the diagnosis of chronic infections. The other supplementary tests, such as the complement fixation (CF) test and ELISA, require special equipment, reagents and trained personnel. The Rose Bengal plate (RBP) test has shown potential as a good rapid diagnostic test. This is a report of serum samples from suspected cases of brucellosis that were tested using the RBP, SA and CF tests. The RBP test was shown to have a better relative sensitivity and as good specificity as the SA when compared with the CF test, and may be a useful initial diagnostic test for hospitals in remote rural areas if properly conducted with well stored antigen.
\end{abstract}

\section{Keywords}

Brucellosis, Human, Diagnosis, Rose Bengal Plate Test

\section{Introduction}

Brucellosis, caused by the bacteria of Brucella genus, is an important zoonotic 
disease with economic and public health implications almost all over the world [1]. Clinical manifestations of brucellosis in humans vary since predominating signs may be related to complications affecting either gastrointestinal, respiratory, osteoarticular, cardiovascular or neurologic systems [2] [3] [4]. This can lead to misdiagnosis of the disease [5]. The most accurate diagnosis of brucellosis is by isolating the organism from infected individuals, but these isolations are infrequent due to the overuse of antibiotics prior to obtaining test material and the lack of facilities and personnel to perform these isolations [6] [7]. Blood culture, the standard method of diagnosis, is only effective during the acute stage of the disease and in practice, the isolation and confirmation of the Brucella organism is difficult and time consuming [8] [9]. Newer techniques that detect either the antigen or the DNA of the Brucella organisms are showing promising results however, they are not commonly used and require further evaluation [10] [11]. Presently, brucellosis is diagnosed by a combination of serological tests, such as the serum agglutination (SA) test, which is still used widely, but has a drawback in that only acute brucellosis with a high or rising antibody titer can be confirmed. In many chronic cases where the SA test gives an equivocal result, either the complement fixation (CF) or the anti-human gamma globulin test (AHG/ Coombs) tests may be more informative. Several countries have adopted the enzyme-linked immunoassay (ELISA) for the serologic diagnosis of the disease in humans and other species. Since isotypes of immunoglobulins induced can be measured, ELISA is useful in indicating the status of infections. Immunoblot that detects antibodies to selected cytoplasmic proteins of Brucella may be useful in differentiating active from past or sub clinical infection. However, both these tests require equipment, reagents and experienced personnel, which may be unavailable in developing countries. Many developing countries, including Kenya, are in an economic flux, which has led to a decline in the laboratory diagnostic services. The Rose Bengal plate (RBP) test is an affordable, quick, simple and efficient screening test [12] [13]. The card test variation of the RBP test was found efficient in diagnosis of the acute human brucellosis and has been suggested for use in the diagnosis of chronic cases [14] [15]. In this paper, we compare the results of the RBP test with that of SA and CF tests on sera obtained from individuals with suspected brucellosis or with pyrexia of unknown origin (PUO).

\section{Materials and Methods}

\subsection{Sources of Sera}

Samples were collected between 1972 and 1980 by clinics and hospitals in specified parts of Kenya (Figure 1). Serum samples were obtained from 965 patients that reported at Machakos General Hospital (MKS) with either one major sign or PUO, hepato-splenomegaly and arthritis, or two minor signs of sweating, headache or extreme weakness. A total of 152 serum samples obtained from individuals attending Siyapei Dispensary, Narok (NAR), with complaints of PUO, joint and back pains were sent to the Veterinary Research Laboratory, 


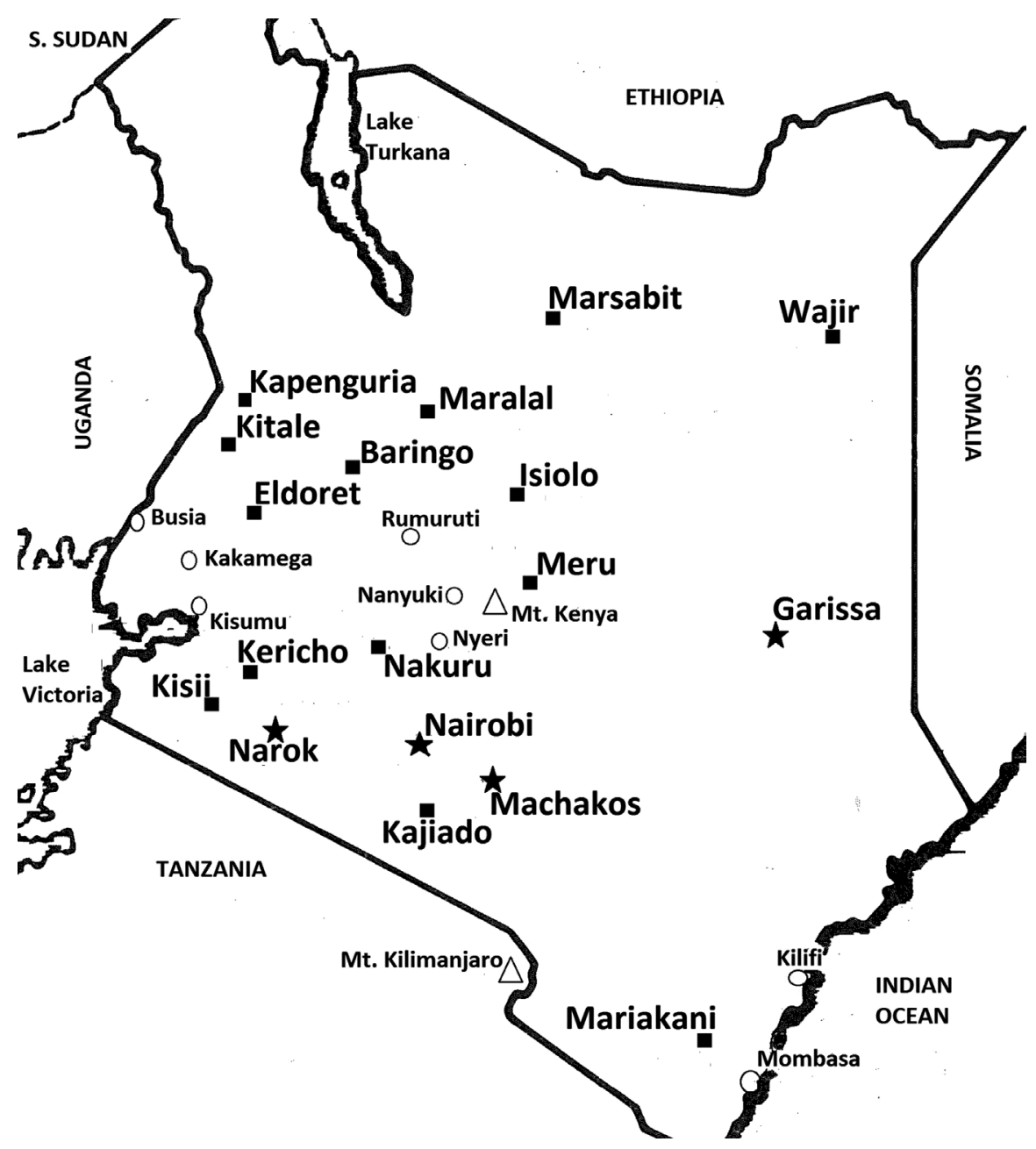

Figure 1. Map of Kenya. Machakos (MKS), Narok (NAR), Nairobi (NBI) and Garissa (GAR) marked with $\star$. Other (OTH) sources marked with $\boldsymbol{\square}$. Names from top (left to right): Marsabit (OTH); Wajir (OTH); Kapenguria (OTH); Kitale, Baringo, Maralal (OTH); Eldoret, Isiolo (OTH); Meru (OTH); Kericho, Nakuru (OTH); Garissa; Kisii (OTH); Narok (NAR), Nairobi (NBI), Machakos (MKS); Kajiado (OTH); Mariakani (OTH).

Kabete, where they were tested with RBP, SA and CF tests. A clinician in a community in Garissa (GAR) sent seventy serum samples from patients with suspected brucellosis based on clinical signs. Over a period of 2 months, a private laboratory in Nairobi (NBI) collected 146 sera from individuals with PUO. The remaining samples $(\mathrm{OTH})$ were from either laboratory personnel routinely tested for brucellosis, veterinarians or individuals with PUO from parts of the country (some of these sites are marked in Figure 1) not included in the above regions.

\subsection{Serological Tests}

The SA, CF and RBP tests were done as described previously [12]. The SA test was interpreted as positive if the titer was $>100$ international units, with the CF test, if the titer was $>1 / 10$ and with the RBP test if agglutination was observed. 


\subsection{Statistical Analysis}

The results were set in a $2 \times 2$ contingency table to compare the sensitivity (probability of false negative) and the specificity (probability of false positive) of the RBP and CF tests relative to the standard SA test [16]. The $\chi^{2}$ values for correlated proportions that are not independent were calculated using McNemar's test [17] to determine the significance of the paired values [18].

\section{Results}

Out of 965 samples submitted from MKS, 200 were not tested in RBP test but only tested in SA and CF tests. The rest, 765 samples, were tested in all the three tests with 79 samples showing a positive reaction in the RBP test (Table 1). Sixty five of these were positive with SA test, of which 47 were also positive with CF test. Two samples positive with CF test were negative with the SA test. Twelve RBP test reactors were negative with these 2 tests. Five samples each were positive with either the SA or CF tests out of 686 samples that had negative reactions with the RBP test.

A total of 118 of 152 samples tested from NAR were positive with the RBP test. Seventy-eight samples of the 118 samples were also positive with both the SA and CF tests. Sixteen samples were positive with SA test and 17 with CF test. Seven of the RBP test positive samples were negative with the SA and CF tests. A total of 33 samples were negative with all the three tests.

Twenty of 70 samples from GAR were positive with RBP test. Only 10 of these were positive with both the SA and CF tests. Five samples of RBP test positive were negative with SA and CF tests. Three of the $20 \mathrm{RBP}$ test positive were positive with CF test but not with SA test, whereas 2 were positive with SA test only. Fifty samples were negative with all the three tests.

From Nairobi, 131 of the 137 samples were negative with the RBP test. Two of these were positive with the CF test and 3 were positive with the SA test. Four of the 6 samples positive with the RBP test were positive with the CF test only and the other 2 were positive with the CF test and the SA test. One of the 2 samples

Table 1. Comparison of Rose Bengal plate (RBP) test positive and RBP test negative sera with serum agglutination (SA) and complement fixation (CF) tests.

\begin{tabular}{ccccccccccccccc}
\hline \multicolumn{1}{c}{ RBP positive } & \multicolumn{1}{c}{ RBP negative } \\
\hline Source* & MKS & NAR & GAR & NBI & OTH & ST & MKS & NAR & GAR & NBI & OTH & ST & GT \\
\hline Test & & & & & & & & & & & & & \\
\hline SA-/CF- & 12 & 7 & 5 & 1 & 5 & $\mathbf{3 0}$ & 676 & 33 & 50 & 126 & 155 & 1041 & 1071 \\
SA-/CF+ & 2 & 17 & 3 & 2 & 3 & 27 & 5 & 1 & 0 & 2 & 1 & 8 & 35 \\
SA+/CF- & 18 & 16 & 2 & 1 & 7 & 44 & 5 & 0 & 0 & 3 & 1 & 9 & 53 \\
SA+/CF+ & 47 & 78 & 10 & 2 & 17 & 154 & 0 & 0 & 0 & 0 & 0 & 0 & 154 \\
TOTAL & 79 & $\mathbf{1 1 8}$ & $\mathbf{2 0}$ & $\mathbf{6}$ & $\mathbf{3 2}$ & $\mathbf{2 5}$ & $\mathbf{6 8 6}$ & $\mathbf{3 4}$ & $\mathbf{5 0}$ & $\mathbf{1 3 1}$ & $\mathbf{1 5 7}$ & $\mathbf{1 0 5 8}$ & $\mathbf{1 3 1 3}$ \\
\hline
\end{tabular}

*MKS-Machakos; NAR-Narok, Gar-Garissa, NBI-Nairobi, OTH-Others (see Figure 1). 
that were negative with the CF test was positive with the SA test.

Thirty two samples out of 189 samples from the other (OTH) sources were positive with the RBP test. Seventeen of these 32 samples were positive with the SA test as well as the CF test. Seven positive with the SA test were negative with the CF test, and 3 positive with the CF test were negative with the SA test. Of the 157 negative with the RBP test, one sample each was positive with either the SA test or the CF test.

The results of 1313 serum samples were used for the analysis of relative sensitivity and specificity of the RBP, SA and CF tests. Out of the 255 samples positive with the RBP test, 154 were positive with both the SA and the CF tests. Thirty of the RBP positives were negative with the SA test as well as the CF test. Twenty-seven were positive with the CF test but negative with the SA test, whereas 44 were positive with the SA test but negative with the CF test. One thousand fifty eight samples were negative with the RBP test, of which 1041 were also negative with the SA and CF tests. Eight and nine samples were positive with either the CF test or the SA test, respectively.

The agreement between the 255 RBP test positive sera was $77.6 \%$ and $71.2 \%$ for SA and CF tests respectively. The agreement to the SA test results varied from $50 \%$ for samples from NBI to $82.3 \%$ for samples from MKS. For the CF test, the agreement ranged from $66.7 \%$ for samples from NBI to $80.5 \%$ for samples from NAR.

The agreement between 1058 RBP test negative sera was $99.1 \%$ and $98.4 \%$ for the SA and CF tests respectively. The agreement for the SA test ranged from $98.5 \%$ for sera from NBI to $100 \%$ for samples from NAR and GAR. The agreement for the CF test ranged from $97.7 \%$ for NBI samples to $100 \%$ for NAR and GAR samples.

The agreement between SA and CF tests for RBP test positives was $91.4 \%$ (ranging from $75.4 \%$ for $\mathrm{MKS}$ to $100 \%$ for $\mathrm{NBI}$ ) and for RBP test negatives was 99.2\% (ranging from $99.3 \%$ for MKS to $100 \%$ for NAR, NBI and GAR).

The relative sensitivities (RSe) and the relative specificities (RSp) of the RBP and CF tests, calculated from Table 1 results, were compared with the results of the SA test, and are shown in Table 2.

The RBP test had a higher RSe (95.6\%) compared to the RSe of the CF test (74.4\%) in relation to the results of SA test. The RSe for the RBP test ranged from $50 \%$ for the NBI samples to $100 \%$ for the NAR and GAR samples. However, RSp of the RBP test was lower (94.8\%) than the CF test (96.8\%), and ranged from $58.6 \%$ for NAR to $\sim 97 \%$ for samples from MKS and NBI. Except for the samples from NBI, the correlation was significantly different between RBP test and SA test ( $\mathrm{p}<0.05$ to $\mathrm{p}<0.01$ ). There was better correlation between CF and SA tests except for the samples from MKS ( $p<0.01)$. The RSe and RSp of the RBP test to CF test was $95.8 \%$ and $93.4 \%$ respectively but there was a significant difference in correlation between the results of these two tests ( $p<0.5$ to $p$ $<0.01$ ), except for NBI samples (Table 3 ). 


\section{Discussion}

Clinicians have to rely on serological tests to confirm the diagnosis of brucellosis in humans since the disease manifests with a variety of symptoms. The SA test is the most used assay, however its results can be confusing where the person does not have the disease but has been repeatedly exposed to an infectious source. Frequently the test is negative or inconclusive during the incubation stage or in the late chronic stage of the disease, since following an infection the IgM declines more rapidly than IgG levels, and in chronic stages of the disease the predominant immunoglobulin present is IgG [14]. The use of anti-human gamma globulin serum (AHG) in the test was found to be more reliable and sensitive in detecting such non-agglutinating antibodies [19]. The CF test has also been found to be more reliable in diagnosing brucellosis. Kerr et al. [20] considered the CF test as a valuable test in detecting antibodies directly associated with the activity of the infection. A good correlation exists between the results of AHG and CF tests [20] and between the results of AHG, CF and RBP tests [14]. However, Oomen and Waghela [12] found the RBP test to give more false positives than the CF test. Araj et al. [21] found that the RBP test, positive in $98 \%$ of the patients with acute brucellosis and in $64 \%$ with chronic brucellosis, and was more sensitive than the SA test, positive in $51 \%$ and $27 \%$ of acute and chronic

Table 2. Relative sensitivity (RSe) and Relative specificity (RSp) of the Rose Bengal plate (RBP) and the Complement fixation (CF) tests in relation to the Serum Agglutination (SA) test.

\begin{tabular}{ccccccc}
\hline & \multicolumn{3}{c}{ RBP } & & \multicolumn{3}{c}{ CF } \\
\hline Source* & RSe\% & RSp\% & $\chi^{2}$ & RSe\% & RSp\% & $\chi^{2}$ \\
\hline MKS & 92.9 & 97.9 & 4.26 & 67.1 & 98.9 & 8.53 \\
NAR & 100.0 & 58.6 & 23.04 & 83.0 & 70.7 & 0.03 \\
GAR & 100.0 & 86.2 & 7.12 & 83.3 & 94.8 & 0.20 \\
NBI & 50.0 & 97.7 & 0.00 & 33.3 & 96.9 & 0.12 \\
OTH & 96.0 & 95.1 & 5.44 & 68.0 & 97.6 & 1.33 \\
TOTAL & $\mathbf{9 5 . 6}$ & $\mathbf{9 4 . 8}$ & $\mathbf{3 4 . 9 0}$ & $\mathbf{7 4 . 4}$ & $\mathbf{9 6 . 8}$ & $\mathbf{3 . 6 8}$ \\
\hline
\end{tabular}

*MKS-Machakos; NAR-Narok, Gar-Garissa, NBI-Nairobi, OTH-Others (see Figure 1). Tabulated $(\mathrm{df}=1)$ $\chi^{2}: \mathrm{p}<0.1=2.707 ; \mathrm{p}<0.05=3.841 ; \mathrm{p}<0.01=6.635$.

Table 3. RSe and RSp of the RBPT compared to CFT.

\begin{tabular}{cccc}
\hline & \multicolumn{2}{l}{ RBP } & \\
\hline Source & RSe\% & RSp\% & $\chi^{2}$ \\
\hline MKS & 90.7 & 95.8 & 17.86 \\
NAR & 98.9 & 57.9 & 21.16 \\
GAR & 100.0 & 87.7 & 6.13 \\
NBI & 66.7 & 98.5 & 0.00 \\
OTH & 95.2 & 92.9 & 9.30 \\
TOTAL & 95.8 & 93.4 & $\mathbf{5 3 . 1 2}$ \\
\hline
\end{tabular}

*MKS-Machakos; NAR-Narok, Gar-Garissa, NBI-Nairobi, OTH-Others (see Figure 1). Tabulated $(\mathrm{df}=1)$ $\chi^{2}: \mathrm{p}<0.1=2.707 ; \mathrm{p}<0.05=3.841 ; \mathrm{p}<0.01=6.635$. 
cases, respectively. The RBP test results were comparable to $98 \%$ of acute and $60 \%$ of chronic cases positive at a serum dilution of $>1: 80$ obtained with the ELISA. However, ELISA was found to be positive in $2 \%$ of all the serum samples tested from normal subjects and patients with other infectious diseases whereas the RBP and SA tests were negative in such individuals [21]. Sirmatel et al. [22] sampled sera of 184 humans diagnosed clinically with either acute or chronic brucellosis and found the SA test with a higher rate of positivity (83.7\%) compared to $61.9 \%$ for both the RBP test and the IgG detecting ELISA. In the sera from healthy control individuals, the RBP test was positive in $25 \%$ of the individuals negative with both the SA test and the ELISA. In patients with Brucella bacteremia, the sensitivity of ELISA in detecting either IgM or IgG was lower than with the SA test, however, when the results of ELISAs measuring IgM and IgG were combined, the test had similar sensitivity and specificity to the SA test [23]. Non-specific activity is considered a problem with the interpretation of ELISA results especially in uninfected individuals who are at risk for Brucella infections [24]. Merta et al. [25] tested, with SA and RBP tests, serum samples from 310 patients with clinical signs, which would evoke a differential diagnosis including brucellosis. Both the tests showed a $100 \%$ specificity and sensitivity compared with culture positive individuals. The RBP test is considered highly sensitive and specific, but it remains positive for long periods of time in patients who have received treatment for brucellosis [15] [26]. Lucero and Bolpe [27] found that the SA test was less sensitive than the RBP test relative to the CF test in 142 individuals with suspected brucellosis, and was less specific in the 307 serum samples from asymptomatic or urban populations since one sample was positive with the SA test while all were negative with the RBP and CF tests. In another study the RBP test detected all the culture positive as well as all the SA test positive individuals. The RBP test had a comparable specificity since none of the 97 individuals which were negative with the SA test was positive with the RBP test. Contrary to this, the RBP test was positive in 7 samples, which were either negative or equivocal with the SA test [28]. In the present study, there was a close agreement between the SA test (99.1\%) and the CF test (98.4\%) in the sera negative with the RBP test. Relative to the diagnosis with the SA test, the RBP test had a higher sensitivity compared with the CF test. The RSe was higher for the samples from individuals who were suspected of having brucellosis or may have been in contact with animals (MKS, NAR, GAR and OTH) compared to the samples from persons with PUO in the urban areas (NBI). However, the RSp of the RBP test with the SA test as reference was lower than RSp of CF test. The source of the serum samples did not affect the specificity of the RBP test. There is a high significant difference in correlation when all the RBP test results are compared to the SA test results $\left(\chi^{2}\right.$ values $\left.=34.90, \mathrm{p}<0.01\right)$. However, this difference in correlation decreases if individual sources of sera are considered $\left(\chi^{2}\right.$ values $=0.0$ to $22.4,0.0<\mathrm{p}<0.01)$. The significant difference in correlation between the SA and CF tests is less when individual source results ( $\chi^{2}$ values $=0.0$ to $1.33, \mathrm{p}>0.1)$ are compared, except for the samples from NAR $\left(\chi^{2}\right.$ values $=3.43$, 
$\mathrm{p}$ <0.05). Thus, the significant difference in correlation is due to the NAR samples. The RSp of RBP test and the CF test is equal to that to the SA test. However, its sensitivity was comparatively lower, $71 \%$ to CF test versus $76 \%$ to the SA test. Clavijo et al. [29] reported that all 87 individuals that had acute $(<3$ months) disease were positive on culture for Brucella, whereas only 23 of 46 that had chronic ( $>3$ months) disease were positive. The RBP test was more sensitive, since out of 133 patients, 128 patients were positive with RBP test compared to 120 and 90 with the SA and IgM detecting ELISA tests, respectively. Dabdoob and Abdulla [30] found the RBP test can be adapted to a titration method, and with one source of RBP antigen the results were not significantly different from the SA test results. The performance of ELISA and CF tests requires facilities, special reagents, equipment and trained personnel. Also, the $\mathrm{CF}$ test may detect IgG, indicative of chronic disease and suffers from anti-complementary activity, which reduces the specificity [31]. In our study, we regarded any serum sample with anti-complementary activity as negative. If we removed all 179 such samples from analysis, the RSe of the CF test improved to $88.3 \%$ but did not match that of RBP test (data not shown). The RBP test offers an inexpensive and practical rapid test, which is simple to perform without requiring special equipment, even when compared to the SA test. It is a useful test for hospitals and clinics in areas, which do not have skilled personnel and where the patients cannot come in for repeat exams. Maichomo et al. [32] found discrepancy in the results of RBP test conducted on 488 serum samples at a field dispensary to the results of the RBP test on sera. Nonetheless, there was good agreement between the SA, CF test and RBP tests conducted at the central laboratory. In the present study, 102 of the 118 sera positive in the RBP test performed at a remote clinic in Narok district of Kenya were positive on repeat test at the central laboratory in Kabete (data not shown). However, a recent study demonstrates a good agreement between RBP test and SA test [33]. The RBP test may be an initial test of choice, if adequate standards of performance of the RBP test and the reagent quality are maintained, in rural hospitals and clinics. This is especially true for developing countries where the population is at a greater risk of infection and clinics are not properly equipped for performing ELISA or CF test [12] [32] [33]. Our study shows that RBP test, a comparatively rapid test, has good sensitivity and specificity relative to the routinely used SA test supporting the finding of Yohannes et al. [34], who recommended use of at least two tests coupled with clinical history of the patient to confirm the diagnosis of brucellosis.

\section{References}

[1] Boschiroli, M.-L., Foulongne, V. and O’Callaghan, D. (2001) Brucellosis: A Worldwide Zoonosis. Current Opinion in Microbiology, 4, 58-64. https://doi.org/10.1016/S1369-5274(00)00165-X

[2] Young, E.J. (1995) An Overview of Human Brucellosis. Clinical Infectious Diseases, 21, 283-289. https://doi.org/10.1093/clinids/21.2.283

[3] Potasman, I., et al. (1991) Brucellosis: An Unusual Diagnosis for a Seronegative 
Patient with Abscesses, Osteomyelitis, and Ulcerative Colitis. Review of Infectious Diseases, 13, 1039-1042. https://doi.org/10.1093/clinids/13.6.1039

[4] Shakir, R.A., et al. (1987) Clinical categories of neurobrucellosis. Brain, 110, 213 223. https://doi.org/10.1093/brain/110.1.213

[5] Mantur, B.G., et al. (2006) Protean Clinical Manifestations and Diagnostic Challenges of Human Brucellosis in Adults: 16 Years' Experience in an Endemic Area. Journal of Medical Microbiology, 55, 897-903. https://doi.org/10.1099/jmm.0.46097-0

[6] Lopez-Merino, A., López Santiago, R. and Madkour, M.M. (1989) Immunology of Brucellosis in Humans.

[7] Smirnova, E., Vasin, A.V., Sandybaev, N., Klotchenko, S., Plotnikova, M., Chervyakova, O., Sansyzbay, A. and Kiselev, O. (2013) Current Methods of Human and Animal Brucellosis Diagnostics. Advances in Infectious Diseases, 3, 177-184. https://doi.org/10.4236/aid.2013.33026

[8] Kolman, S., et al. (1991) Comparison of the Bactec and Lysis Concentration Methods for Recovery of Brucella Species from Clinical Specimens. European Journal of Clinical Microbiology and Infectious Diseases, 10, 647-648. https://doi.org/10.1007/BF01975817

[9] Solomon, H.M. and Jackson, D. (1992) Rapid Diagnosis of Brucella melitensis in Blood: Some Operational Characteristics of the BACT/ALERT. Journal of Clinical Microbiology, 30, 222-224.

[10] Matar, G.M., Khneisser, I.A. and Abdelnoor, A.M. (1996) Rapid Laboratory Confirmation of Human Brucellosis by PCR Analysis of a Target Sequence on the 31-Kilodalton Brucella antigen DNA. Journal of Clinical Microbiology, 34, 477-478.

[11] Al Dahouk, S., et al. (2003) Laboratory-Based Diagnosis of Brucellosis-A Review of the Literature. Part I: Techniques for Direct Detection and Identification of Brucella spp. Clinical Laboratory, 49, 487.

[12] Oomen, L.J. and Waghela, S. (1974) The Rose Bengal Plate Test in Human Brucellosis. Tropical and Geographical Medicine, 26, 300-302.

[13] Feinhaken, D., Schneersonporat, S. and Tamarin, R. (1979) Serological Findings and Their Relevance in Diagnosis of Brucellosis in General Population and in Veterinary Personnel. Refuah Veterinarith, 36, 96-103.

[14] Morgan, W.J., et al. (1969) The Rose Bengal Plate Agglutination Test in the Diagnosis of Brucellosis. Veterinary Record, 85, 636-641.

[15] Diaz, R. and Moriyón, I. (1989) Laboratory Techniques in the Diagnosis of Human Brucellosis. In: Young, E.J. and Corbel, M.J., Eds., Brucellosis. Clinical and Laboratory Aspect, CRC Press, Boca Raton, 73-83.

[16] Martin, S.W. (1977) The Evaluation of Tests. Canadian Journal of Comparative Medicine, 41, 19.

[17] Snedecor, G.W. and Cochran, W.G. (1971) Méthodes Statistiques, Original Title: Statistical Methods. 6th Edition, Iowa State University Press, Ames, 649.

[18] Sard, D.M. (1978) Dealing with Data: The Practical Use of Numerical Information-(8) Expectation and Observation. Veterinary Record, 103, 552-555. https://doi.org/10.1136/vr.103.25.552

[19] Kerr, W.R., et al. (1967) Immunoglobulin Class of Brucella Antibodies in Human Sera. Immunology, 13, 223.

[20] Kerr, W.R., et al. (1966) The Laboratory Diagnosis of Chronic Brucellosis. The Lancet, 288, 1181-1183. https://doi.org/10.1016/S0140-6736(66)90492-2 
[21] Araj, G., et al. (1986) Evaluation of Elisa in the Diagnosis of Acute and Chronic Brucellosis in Human Beings. Epidemiology and Infection, 97, 457-469. https://doi.org/10.1017/s0022172400063634

[22] Sirmatel, F., Türker, M. and Bozkurt, A.I. (2002) Evaluation of the Methods Used for the Serologic Diagnosis of Brucellosis. Mikrobiyoloji Bulteni, 36, 161-167.

[23] Memish, Z., et al. (2002) Comparison of the Brucella Standard Agglutination Test with the ELISA IgG and IgM in Patients with Brucella bacteremia. Diagnostic Microbiology and Infectious Disease, 44, 129-132. https://doi.org/10.1016/S0732-8893(02)00426-1

[24] Heizmann, W., et al. (1985) Brucellosis: Serological Methods Compared. Journal of Hygiene, 95, 639-653. https://doi.org/10.1017/S0022172400060745

[25] Mert, A., et al. (2003) The Sensitivity and Specificity of Brucella Agglutination Tests. Diagnostic Microbiology and Infectious Disease, 46, 241-243. https://doi.org/10.1016/S0732-8893(03)00081-6

[26] Awad, R. (1998) Human Brucellosis in the Gaza Strip, Palestine. Eastern Mediterranean Health Journal, 4, 225-233.

[27] Lucero, N.E. and Bolpe, J.E. (1998) Buffered Plate Antigen Test as a Screening Test for Diagnosis of Human Brucellosis. Journal of Clinical Microbiology, 36, 1425 1427.

[28] Altwegg, M. and Bohl, E. (1985) Evaluation of a Rapid, Reliable, and Inexpensive Screening Test for the Serological Diagnosis of Human Brucellosis. Zentralblatt für Bakteriologie, Mikrobiologie und Hygiene. Series A: Medical Microbiology, Infectious Diseases, Virology, Parasitology, 260, 65-70. https://doi.org/10.1016/S0176-6724(85)80099-7

[29] Clavijo, E., et al. (2003) Comparison of a Dipstick Assay for Detection of BrucellaSpecific Immunoglobulin M Antibodies with Other Tests for Serodiagnosis of $\mathrm{Hu}$ man Brucellosis. Clinical and Diagnostic Laboratory Immunology, 10, 612-615.

[30] Dabdoob, W. and Abdulla, Z. (2000) A Panel of Eight Tests in the Serodiagnosis and Immunological Evaluation of Acute Brucellosis. Eastern Mediterranean Health Journal, 6, 304-312.

[31] Lucero, N.E., et al. (2007) The Value of Serologic Tests for Diagnosis and Follow up of Patients Having Brucellosis. American Journal of Infectious Diseases, 3, 27-35. https://doi.org/10.3844/ajidsp.2007.27.35

[32] Maichomo, M., et al. (1998) Assessment of the Rose-Bengal Plate Test for the Diagnosis of Human Brucellosis in Health Facilities in Narok District, Kenya. East African Medical Journal, 75, 219.

[33] Glanville, W.A., et al. (2017) Poor Performance of the Rapid Test for Human Brucellosis in Health Facilities in Kenya. PLOS Neglected Tropical Diseases, 11, e0005508. https://doi.org/10.1371/journal.pntd.0005508

[34] Yohannes, M., et al. (2012) Comparative Evaluation of the Rose Bengal Plate Test, Standard Tube Agglutination Test and Complement Fixation Test for the Diagnosis of Human Brucellosis. Revue Scientifique et Technique (International Office of Epizootics), 31, 979-984. https://doi.org/10.20506/rst.31.3.2175 
Submit or recommend next manuscript to SCIRP and we will provide best service for you:

Accepting pre-submission inquiries through Email, Facebook, LinkedIn, Twitter, etc. A wide selection of journals (inclusive of 9 subjects, more than 200 journals)

Providing 24-hour high-quality service

User-friendly online submission system

Fair and swift peer-review system

Efficient typesetting and proofreading procedure

Display of the result of downloads and visits, as well as the number of cited articles Maximum dissemination of your research work

Submit your manuscript at: http://papersubmission.scirp.org/

Or contact ojcd@scirp.org 